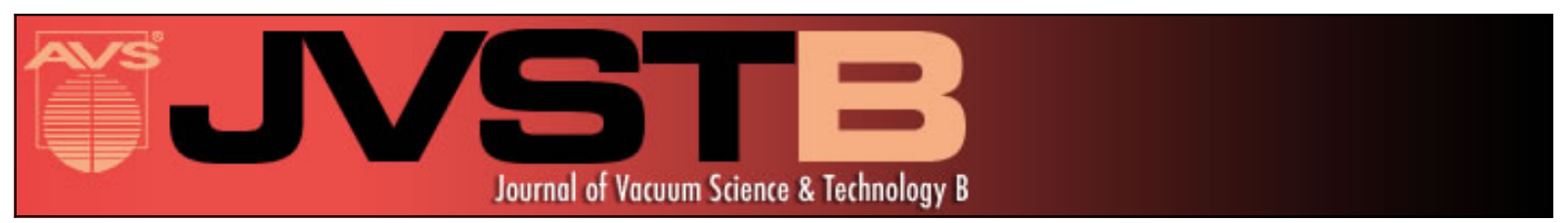

\title{
Control of edge bulge evolution during photoresist reflow and its application to diamond microlens fabrication
}

Hangyu Liu, Johannes Herrnsdorf, Erdan Gu, and Martin D. Dawson

Citation: Journal of Vacuum Science \& Technology B 34, 021602 (2016); doi: 10.1116/1.4943558

View online: http://dx.doi.org/10.1116/1.4943558

View Table of Contents: http://scitation.aip.org/content/avs/journal/jvstb/34/2?ver=pdfcov

Published by the AVS: Science \& Technology of Materials, Interfaces, and Processing

\section{Articles you may be interested in}

Fabrication of polydimethylsiloxane microlens arrays on a plastic film by proton beam writing

J. Vac. Sci. Technol. B 32, 06F506 (2014); 10.1116/1.4900419

Fabrication of bioinspired omnidirectional and gapless microlens array for wide field-of-view detections Appl. Phys. Lett. 100, 133701 (2012); 10.1063/1.3696019

Complete reversal imprinting for fabricating microlens arrays with faithful shape replication

J. Vac. Sci. Technol. B 27, 2781 (2009); 10.1116/1.3258150

Fabrication of natural diamond microlenses by plasma etching

J. Vac. Sci. Technol. B 23, 130 (2005); 10.1116/1.1843826

Reflection/transmission confocal microscopy characterization of single-crystal diamond microlens arrays Appl. Phys. Lett. 84, 2754 (2004); 10.1063/1.1695101

\section{FIDEN}

\section{Instruments for Advanced Science}

w www.HidenAnalytical.com E info@hiden.co.uk

CLICK TO VIEW our product catalogue

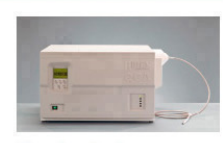

Gas Analysis

dynamic measurement of reaction gas streams catalysis and thermal analysis

molecular beam studies

fermentation environmental and ecological studies

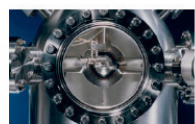

Surface Science

, UHVTPD

SIMS elemental imaging - surface mapping

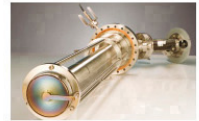

Plasma Diagnostics plasma source characterization etch and deposition process reaction , analysis of neutral and radical species

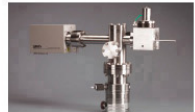

Vacuum Analysis partial pressure measurement and control of process gases reactive sputter process control vacuum diagnostics , 


\title{
Control of edge bulge evolution during photoresist reflow and its application to diamond microlens fabrication
}

\author{
Hangyu Liu, ${ }^{\text {a) }}$ Johannes Herrnsdorf, ${ }^{\text {a) }}$ Erdan Gu, ${ }^{\text {b) }}$ and Martin D. Dawson \\ Department of Physics, Technology and Innovation Centre, Institute of Photonics, University of Strathclyde, \\ 99 George Street, Glasgow G1 IRD, United Kingdom
}

(Received 25 November 2015; accepted 22 February 2016; published 9 March 2016)

\begin{abstract}
The authors present an empirical study of profile evolution of lithographically defined photoresist (PR) patterns during thermal reflow and apply the findings to diamond microlens fabrication. During PR reflow, a bulge forms at the edge of the PR pattern and propagates inwards as the temperature and PR thickness are increased. An empirical relationship for this propagation is derived. Furthermore, it was found that at a certain reflow temperature and a limited pattern size, there is a minimum initial thickness of the PR pattern for forming spherical lens profiles. Based on these findings, diamond microlenses with a diameter of $400 \mu \mathrm{m}$ and a previously unachieved radius of curvature of over $13 \mathrm{~mm}$ were fabricated. This is underpinned by forming PR microlens patterns with a large radius of curvature and transferring the PR patterns through low-selectivity $\mathrm{Ar} / \mathrm{Cl}_{2}$ inductively coupled plasma etching. (C) 2016 American Vacuum Society.

[http://dx.doi.org/10.1116/1.4943558]
\end{abstract}

\section{INTRODUCTION}

Thermal reflow is a commonly used technique to fabricate microlens features in the photoresist (PR). ${ }^{1}$ These PR microlenses can be transferred onto other materials by etching to form convex lenses ${ }^{2}$ or by molding to form concave lenses ${ }^{3}$ on different substrates, such as GaN, sapphire, and diamond. These microlenses have a broad range of applications in optoelectronics and laser optics. ${ }^{4-7}$ Our work here is particularly motivated by the pattern transfer into diamond, which has excellent properties for photonic applications, such as transmittance over a broad spectral range ${ }^{8}$ and high Raman gain. ${ }^{9}$

Diamond microlenses attract much attention due to their extraordinary performances, e.g., as a laser cavity to stabilize laser oscillation ${ }^{7}$ or as a solid immersion lens to guide light. ${ }^{10,11}$ Especially, a diamond microlens with a large radius of curvature (ROC) is desired in semiconductor disk lasers and diamond Raman lasers to provide mode matching or reduce the risk of mirror coating damage. ${ }^{12-14}$

Up until now, microlenses are typically fabricated by thermal reflow of a single PR layer ${ }^{15}$ and subsequent pattern transfer by inductively coupled plasma (ICP) etching. This approach is attractive due to its simplicity, high reproducibility, and the high surface quality of the resulting microlenses. ${ }^{2,16}$ The engineering aspects of this particular method have been studied and extensively reported in the recent past. ${ }^{17-19}$ However, even though the PR reflow step plays a crucial role, only little is known about the detailed mechanisms that determine the exact shape of the reflowed PR. A controllable way of fabricating PR lenses with different dimensions and ROCs has not been reported yet.

In this work, we report a detailed empirical study of the profile evolution of lithographically defined photoresist patterns during thermal reflow and explore the mechanisms that

\footnotetext{
${ }^{a)} \mathrm{H}$. Liu and J. Herrnsdorf contributed equally to this work.

b)Electronic mail: erdan.gu@strath.ac.uk
}

govern the shape of the reflowed PR. It is then illustrated how these findings are applied to achieve the state-of-the-art large ROC diamond microlenses. The PR reflow examination carried out in this work is also applicable to fabricate microlenses on other materials.

Importantly, we observe the formation and evolution of a bulge at the edge of lithographically defined PR patterns upon thermal reflow. We refer to this as the "edge bulge effect." To our knowledge, this effect has not been investigated in depth before. There are a couple of known bulge formations that at first may appear similar, namely, the "coffee stain effect" in liquid droplets upon solvent evaporation, ${ }^{20,21}$ the "edge bead effect" in spin coating, ${ }^{22}$ and the bulges associated with moving contact lines ${ }^{23}$ during PR deposition. However, the edge bulge effect reported here is distinctly different from these known effects. We examine the evolution of the edge bulge as a function of the reflow temperature and the initial thickness of the PR patterns and derive an empirical formula describing the relationship. Based on this examination, a controllable fabrication of PR patterns is realized via the reflow method.

Additionally, a previously unreported minimum thickness at a certain reflow temperature for the formation of a convex reflowed microlens shape on PR patterns is observed and characterized. It was further shown that the PR microlens formed at this minimum initial thickness has the largest ROC at its diameter. Based on the study, a PR microlens pattern with the largest reported ROC of $1.4 \mathrm{~mm}$ at a diameter of $400 \mu \mathrm{m}$ is achieved.

Furthermore, a controllable diamond microlens fabrication process is developed based on the investigation of PR reflow and the ICP etching with a low etching selectivity. The process developed enables the realization of high quality diamond microlenses with a ROC $>10 \mathrm{~mm}$, which has not been achieved before. ${ }^{7,12,24,25}$ As a result, single crystal diamond microlenses with a diameter of $400 \mu \mathrm{m}$ and a stateof-the-art ROC of over $13 \mathrm{~mm}$ have been realized. 


\section{FORMATION AND EVOLUTION OF AN EDGE BULGE DURING THE PR REFLOW}

We first provide an in-depth empirical investigation of the PR edge bulge effect and how it applies to reflowed PR microlens patterns with a large ROC. This study gives valuable guidelines for the fabrication of devices based on this technique.

The PR reflow process employed here consists of three steps: the deposition of the PR (Shipley 220-7.0) on the substrate by spin-coating, the definition of the patterns by photolithography, and finally, the PR reflow by heating on a hotplate above its glass transition temperature $T_{g}$ over 3 min. To pattern the sample, the Shipley $220-7.0$ positive PR was first spin coated on the surface of a substrate using a standard spin speed of $3000 \mathrm{rpm}$ for $1 \mathrm{~min}$. Then, the sample was soft baked on a hotplate under a temperature of $90^{\circ} \mathrm{C}$ for $1 \mathrm{~min}$ followed by a 35-s exposure under a Karl Suss Mask Aligner 6 and then developed in PR developer (MicroDev from Microchem) for $1 \mathrm{~min}$. Finally, the sample was cleaned with deionized water and blow dried with a nitrogen gun. Different initial thicknesses of the PR patterns were achieved by varying the spin speed as well as the number of deposited PR layers. PR with number of deposited layers from one to three were deposited by repeating the spin coating process. During the thermal reflow step, the PR turns into a liquid with a fixed contact line and varying contact angle. The typical view is that the PR droplet can be described as an ideal liquid whose shape is determined by the surface energy, the PR volume, and the contact line. ${ }^{26}$ However, the sheer existence of an edge bulge here shows that this simplified view is not always applicable. It is worth pointing out that for reflow times longer than 3 min, PR shapes were observed to be independent of the reflow time and the repetition of the reflow step. The profiles of the patterned PR after reflow and cooling down to room temperature were recorded using a Dektak 3 surface profilometer. In this section, $2 \times 2 \mathrm{~cm}^{2}$ silicon substrates diced from a 6-in. wafer were used which provided a large sample area. However, a similar behavior of PR reflow was also observed on the diamond.

\section{A. Edge bulge observation and evolution}

A schematic illustration of the edge bulge effect is shown in Fig. 1. The curve with arrows represents a profile with edge bulges. As the reflow temperature and/or PR thickness is increased, the bulge propagates inwards as indicated by the arrows until eventually a convex lens of approximately the spherical shape (spherical curve) is formed. The edge bulge propagation is first investigated on large $1 \times 1 \mathrm{~mm}^{2}$ square PR patterns, which minimizes the interaction between the bulges.

A set of micrographs of the propagation process with increasing temperatures at a PR initial thickness of $15 \mu \mathrm{m}$ during thermal reflow is shown in Fig. 2. As can be seen, the edge bulge forms first at the borders of the pattern and then propagates inward when the reflow temperature is gradually increased. We use the propagation distance of the edge bulge $\mathrm{d}_{\max }$, which corresponds to the highest point of the edge

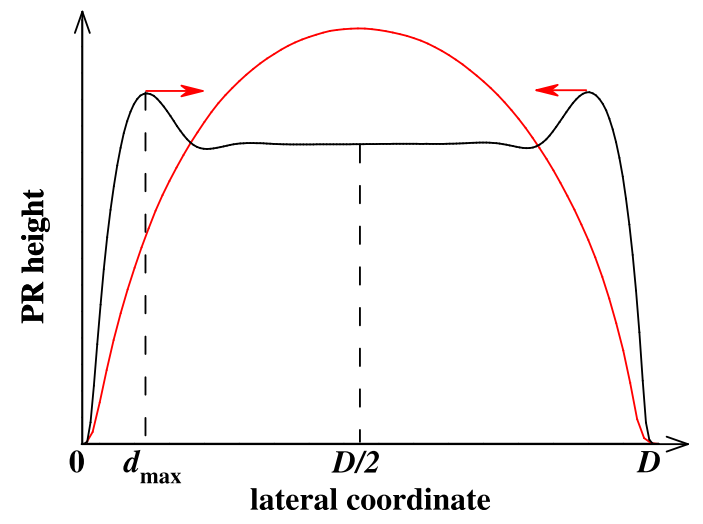

FIG. 1. (Color online) Schematic of the edge bulge effect: the curve with arrows represents a profile with an edge bulge and illustrates the key parameters $\mathrm{d}_{\max }$ and $D$. The spherical curve represents the profile of a convex lens.

bulge, to monitor the propagation quantitatively as illustrated in Fig. 1. Due to the geometry of the Dektak tip with a tip radius of $12.5 \mu \mathrm{m}, \mathrm{d}_{\max }$ is systematically overestimated. All measurements are affected in the same way, and therefore, the trends derived here are reliable and qualitatively confirmed by the micrographs in Fig. 2. The evolution of the edge bulge is characterized as a function of the reflow temperature, $\mathrm{T}$, and the initial thickness of the PR pattern, $\mathrm{h}_{0}$, a)

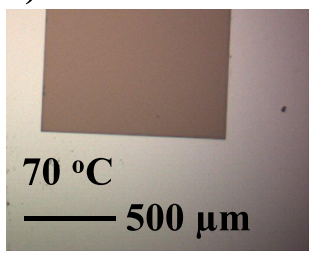

c)

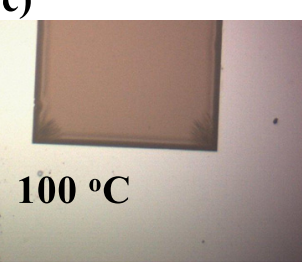

e)

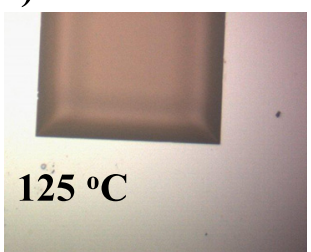

g)

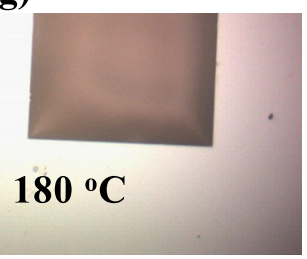

b)

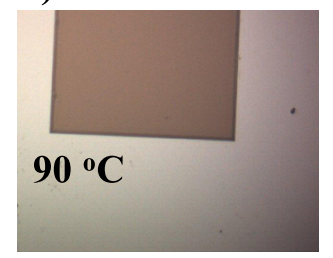

d)

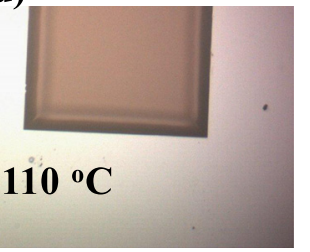

f)

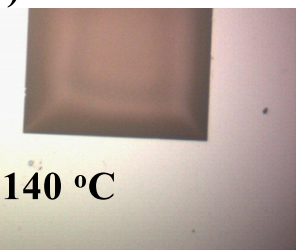

h)

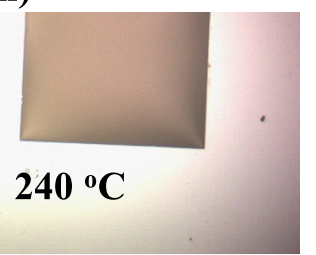

FIG. 2. (Color online) Micrographs of PR patterns $\left(\mathrm{h}_{0}=15 \mu \mathrm{m}\right)$ with different reflow temperatures. (a) Sample before reflow, $\mathrm{T}=70^{\circ} \mathrm{C}$, which is below the PR glass transition temperature. (b)-(h) Evolution of the PR pattern while the temperature was gradually raised to $240{ }^{\circ} \mathrm{C}$. 
before reflow. It was observed that in the case of a circular PR pattern with a diameter $\mathrm{D}$, a convex lens shape is formed when $\mathrm{d}_{\max }=\mathrm{D} / 2$.

To investigate the influences of $\mathrm{T}$ and $\mathrm{h}_{0}$, PR patterns with $\mathrm{h}_{0}$ of $6.3,15$, and $21 \mu \mathrm{m}$, corresponding to the thicknesses of the single-, double-, and three-layer PR deposition, respectively, were reflowed at different temperatures. The resulting profiles were measured. In addition, it was found that a PR thickness $\mathrm{h}_{0}$ around $12 \mu \mathrm{m}$, corresponding to the thinnest achievable PR thickness using double-layer PR deposition, could give adequate uniformity and fidelity for diamond lens fabrication. Thus, a PR pattern reflowed with $\mathrm{h}_{0}$ at $11.6 \mu \mathrm{m}$ was also prepared and measured. The $\mathrm{d}_{\max }$ of these reflowed PR patterns was found to be a function of the reflow temperature. This function can be empirically derived from Fig. 3, which plots $d_{\max }$ vs $T-T_{g}$. The linear theory fit visible in this graph suggests the following empirical relationship:

$$
\mathrm{d}_{\max }=\mathrm{h}_{0} \times\left(\left(\mathrm{T}-\mathrm{T}_{\mathrm{g}}\right) / \mathrm{T}_{\mathrm{p}}\right)^{1 / 3},
$$

where $\mathrm{T}_{\mathrm{p}} \approx 26.6 \times 10^{-3} \mathrm{~K}$ is an empirically determined propagation constant, $\mathrm{h}_{0}$ denotes the initial thickness of the $\mathrm{PR}$ pattern, and $\mathrm{T}_{\mathrm{g}} \approx 352.2 \mathrm{~K}$ denotes the $\mathrm{PR}$ glass transition temperature. The values for $T_{p}$ and $T_{g}$ were obtained by fitting the data shown in Fig. 3.

As can be seen from Eq. (1), the $d_{\max }$ follows a linear relation of $h_{0}$ at a constant reflow temperature. This equation could be used for the estimation of the propagation of a single bulge on the PR after reflow.

\section{B. Bulge interaction and minimum thickness for microlens formation}

In Sec. II A, the undisturbed propagation of a single bulge reflowed on the PR pattern was examined. However, in the specific case of microlens fabrication, the bulges from opposing edges of the pattern will eventually interact and merge to form a lens shape, as shown in Fig. 1. Besides, the bulge propagation might be distorted by the interaction of each other due to a limited propagation area and a circular

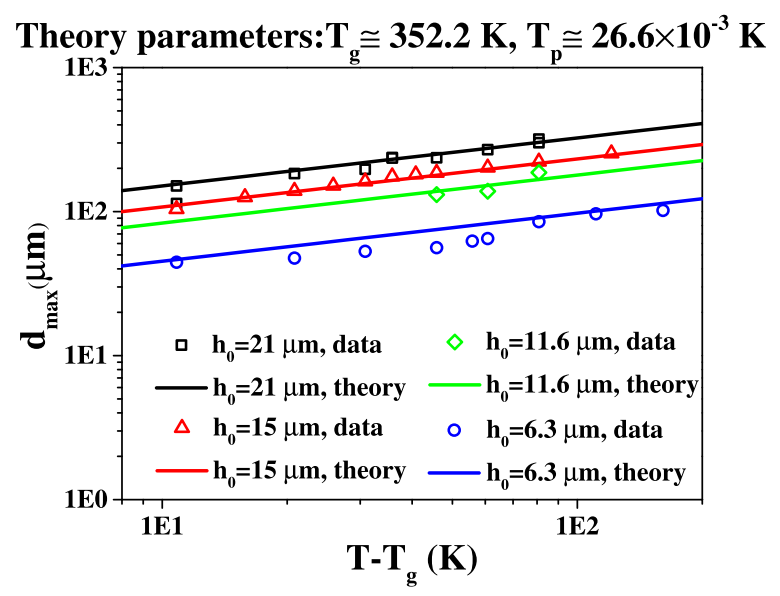

FIG. 3. (Color online) $d_{\max }$ as a function of $\mathrm{T}^{-\mathrm{T}_{\mathrm{g}}}$ with $\mathrm{h}_{0}$ of $6.3,11.6,15$, and $21 \mu \mathrm{m}$, respectively. boundary. This might result in deviations from the propagation behavior described above in Sec. II A. Moreover, a thresholdlike phenomenon occurring at a certain initial thickness $h_{0}$, reflow temperature $T$, and diameter $\mathrm{D}$ is expected when the bulges start to merge. After the bulges completely merged, a convex lens shape is formed as described above.

To investigate the bulge propagation behavior at a limited area, the circular PR patterns with diameters of 200, 250, 350 , and $400 \mu \mathrm{m}$ and different initial thicknesses were fabricated. These PR patterns were then reflowed at a fixed temperature of $125^{\circ} \mathrm{C}$ for $3 \mathrm{~min}$, which are widely used reflow conditions. The $\mathrm{d}_{\max }$ was measured and plotted as a function of $\mathrm{h}_{0}$ in Fig. 4. Note that for $\mathrm{D}<250 \mu \mathrm{m}$, the systematic error of the Dektak measurement leads to a noticeable overestimation of the diameter in the Dektak profile, which is clearly visible in the trace for $\mathrm{D}=200 \mu \mathrm{m}$. An optical image of PR patterns at an initial thickness of $9.15 \mu \mathrm{m}$ before reflow is shown as an inset of Fig. 4. It can be seen that at first, for $\mathrm{h}_{0}<9 \mu \mathrm{m}$, the propagation distance $\mathrm{d}_{\max }$ of the edge bulge is less than that predicted by Eq. (1) as represented by a thick red dashed-dotted line in Fig. 4. As mentioned, this might be an effect of the bulge interaction and the repulsion of opposing edge bulges. Upon further increase in $\mathrm{h}_{0}, \mathrm{~d}_{\max }$ drastically increases, which may be due to the interaction and merging of the bulges.

Additionally, in Fig. 4, the minimum $\mathrm{h}_{0}$ at each diameter indicated by the vertical dashed-dotted lines, which corresponds to the point where the $d_{\max }$ reaches the maximum possible value of the $\mathrm{D} / 2$, can be clearly observed. Above this minimum thickness, the $\mathrm{d}_{\max }$ ceased to increase since the bulges completely merged at the center of the pattern and no further inwards propagation is possible. It is worth pointing out that this minimum thickness is consistent with the thickness given by Eq. (1) for a propagation distance of $d_{\max }=D / 2$. This indicates that even for PR patterns with a limited size, Eq. (1) is applicable to predict the required minimum thickness for bulges merging at the pattern center to form a convex lens shape at a certain reflow temperature T. For example, if we want to achieve a

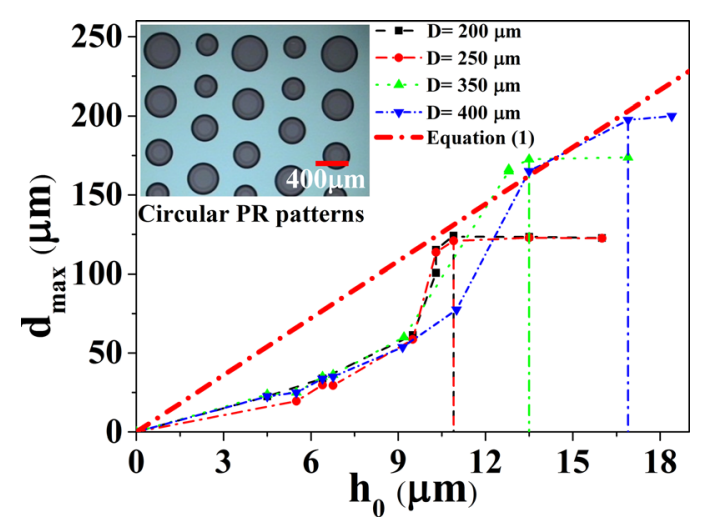

FIG. 4. (Color online) Propagation distance $d_{\max }$ as a function of the initial thickness $h_{0}$ of PR patterns with different diameters. The thick dasheddotted line represents Eq. (1), and the vertical dashed-dotted lines indicate the minimum $h_{0}$ at each diameter. Inset: micrograph of PR patterns with an initial thickness of $9.15 \mu \mathrm{m}$ before thermal reflow at $125^{\circ} \mathrm{C}$. 
convex microlens with a diameter of $\mathrm{D}=400 \mu \mathrm{m}$, the corresponding $\mathrm{d}_{\max }$ should be $200 \mu \mathrm{m}$. For a conventional reflow temperature of $125^{\circ} \mathrm{C}$, the minimum PR thickness is calculated to be $16.68 \mu \mathrm{m}$, which means the PR thickness has to be above this value to form a lens shape after reflow.

In Fig. 5, the minimum PR thickness required for lens shape formation with a given lens radius at a reflow temperature of $125^{\circ} \mathrm{C}$ on silicon is plotted. Similar behavior has also been observed for PR patterns on diamond. Therefore, the above finding can be used as a guideline for the fabrication of PR microlenses on diamond as well.

Furthermore, it was found that the PR lens shape formed at the minimum initial thickness had the largest ROC at its diameter. Based on this result, a PR microlens pattern with the largest reported ROC of $1.4 \mathrm{~mm}$ at a diameter of $400 \mu \mathrm{m}$ is achieved. As shown in Sec. III, such PR microlens pattern with the largest ROC has been successfully transferred onto diamond to form large ROC diamond microlenses.

To quantify how closely the reflowed PR shapes resemble a spherical lens shape, the same set of data as shown in Fig. 4 was fitted by a least squares fit to a spherical shape. As a figure of merit, we use a normalized least squares residual (Res), which is calculated as

$$
\operatorname{Res}=\sum_{i=1}^{N}\left(\left(\mathrm{~h}_{\exp , \mathrm{i}}-\mathrm{h}_{\mathrm{fit}, \mathrm{i}}\right)^{2}\right) /\left(\mathrm{N} \times \mathrm{h}_{0}^{2}\right),
$$

where $h_{\exp }$ is the height of the experimental trace and $h_{\text {fit }}$ is the height of the fitted spherical shape at each point of the trace. The sum is evaluated over all $\mathrm{N}$ points of the trace within the interval $[-\mathrm{D} / 2,+\mathrm{D} / 2]$, in which the traces are centered to zero. The calculation results are plotted in Fig. 6(a). Two examples of the measured and fitted traces are shown in Fig. 6(b). It can be seen that the trace with edge bulges deviates strongly from the fitted microlens shape and this deviation from the measured trace to the fit can therefore be used as a measure for how well the reflowed pattern resembles a spherical lens. In Fig. 6(a), for thicknesses $\mathrm{h}_{0} \leq 9 \mu \mathrm{m}$, Res is approximately constant for different PR

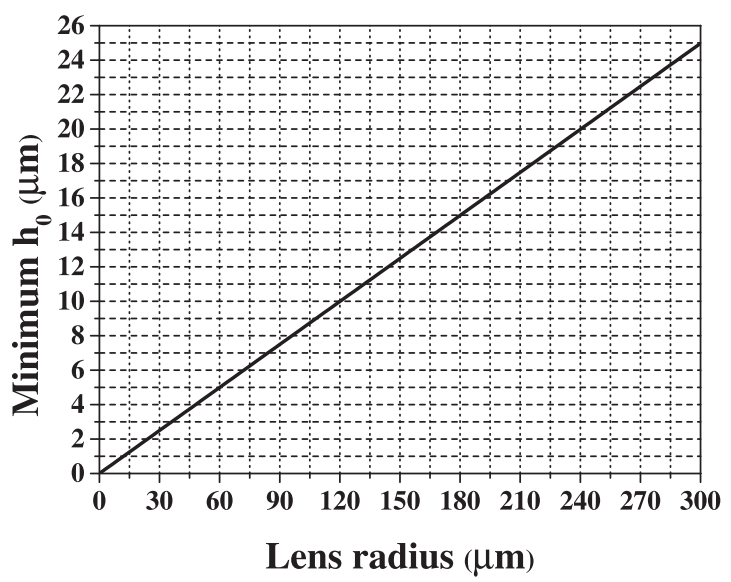

FIG. 5. Minimum PR thickness needed to form a spherical lens shape with a given radius at a reflow temperature of $125^{\circ} \mathrm{C}$ on silicon. thicknesses at a value of $\operatorname{Res} \approx 0.05$. This value corresponds to trace (1) in Fig. 6(b). When $\mathrm{h}_{0}>9 \mu \mathrm{m}$, Res decreases at an exponential rate as indicated by the dashed-dotted line in Fig. 6(a). This means that, once a certain thickness is exceeded, the reflowed PR pattern changes to a spherical lens shape exponentially fast upon further increase in the PR thickness.

Furthermore, these results on the evolution of the PR profile also show that apart from spherical lenses, PR shapes like aspheric lenses or concave rings can also be, in principle, achieved by the precise control of $\mathrm{h}_{0}$ and $\mathrm{T}$.

\section{Discussion}

In this section, we relate our observation of the edge bulge effect to past attempts of understanding the profiles generated by PR reflow on a theoretical basis. In the simplest and most commonly used case, it is assumed that during reflow, the PR becomes an ideal liquid that forms a droplet with a fixed contact line ${ }^{23}$ (or, in a few cases, with a fixed contact angle ${ }^{26}$ ). This approach satisfactorily describes the reflowed PR shapes obtained well above the minimum thickness described in Sec. II B. The more complex shapes observed below minimum thickness, however, cannot be explained by this approach. The underlying reason is that the droplet shape is calculated assuming that the pressure drop calculated by the Young-Laplace law equals a constant term (related to the surface tension) plus a contribution from an external force, i.e., in particular, gravity ${ }^{19}$

$$
1 / \mathrm{R}_{1}+1 / \mathrm{R}_{2}=\mathrm{c}+\mathrm{f}_{\mathrm{ext}},
$$

where $R_{1}$ and $R_{2}$ are the local radii of curvature of the surface, $c$ is a constant, and $f_{\text {ext }}$ is the contribution of external forces. ${ }^{27}$ At the edge bulge, we encounter a change in sign of the local radius of curvature, and thus of the pressure drop calculated by the Young-Laplace law. This intriguing observation is a fundamental problem for this simple approach.

Very few theoretical investigations have been made that consider more complex shapes. Audran et al. ${ }^{18}$ numerically solved the Navier-Stokes equations and were thus able to model the temporal evolution of the droplet, including intermediate shapes that contain an edge bulge. However, their final shape still does not have an edge bulge. Since the edge bulge observed by us does not represent an intermediate temporal shape, it is questionable if it can really be explained by this model. O'Neill and Sheridan ${ }^{17,28}$ summarized a number of fitting procedures for the recorded pattern profiles that account for complex surface shapes. While these fitting methods may be useful for engineering purposes, they do not give any insight into the physics causing the observed shapes.

All in all, the physics of the edge bulge formation are essentially unknown. It is possible that the underlying mechanism is a so far unexplored aspect of fluid mechanics. Here, we mainly focus on the empirical results and its application on the guidance of microlens fabrication. The dynamics behind is still open for discussion. 

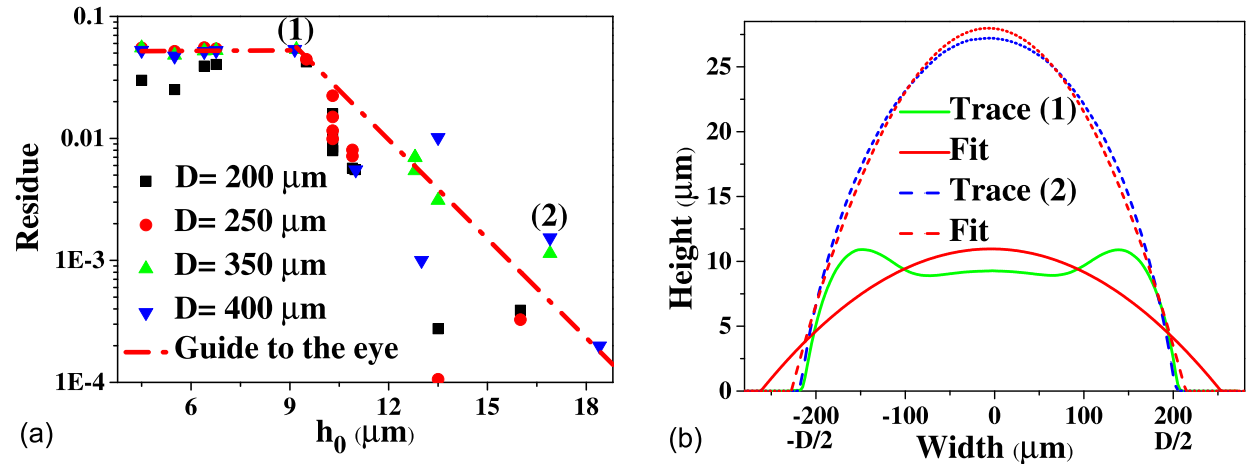

Fig. 6. (Color online) (a) Normalized deviation from a spherical lens shape calculated by Eq. (2) and (b) example traces of measurement and fit of data points (1) and (2) of $\mathrm{D}=400 \mu \mathrm{m}$ shown in (a), trace (1) $\mathrm{h}_{0}=9.15 \mu \mathrm{m}$, Res $=0.054$ and trace (2) $\mathrm{h}_{0}=16.9 \mu \mathrm{m}$, Res $=0.0015$.

\section{FABRICATION OF DIAMOND MICROLENSES WITH A LARGE ROC}

To fabricate diamond microlenses with a large ROC, a lens shape PR structure (as a sacrificial etch mask) with a large ROC and a low ICP etching selectivity for pattern transfer are necessary. The study in Sec. II demonstrates that at a certain diameter, there is a minimum PR thickness required to form a spherical PR lens structure by thermal reflow. The PR lens structure formed by adopting this minimum PR thickness has the largest ROC at a certain diameter as it gives a minimum lens height after thermal reflow. The lens ROC is calculated by Eq. (4)

$$
\mathrm{ROC}=\left(\mathrm{D}^{2}+4 \mathrm{~h}^{2}\right) / 8 \mathrm{~h},
$$

where $\mathrm{D}$ is the lens diameter and $\mathrm{h}$ is the lens height, respectively.

By applying the findings of Sec. II together with a suitable etch method, here, we realized the fabrication of large ROC microlenses on a high quality single crystal diamond. As a result, diamond microlenses with the largest reported ROC of over $13 \mathrm{~mm}$ at a diameter of $400 \mu \mathrm{m}$ are achieved.

\section{A. Fabrication of diamond microlenses}

In this work, a PR microlens array mask with a diameter of $400 \mu \mathrm{m}$ was first fabricated on a $4 \times 4 \mathrm{~mm}^{2}$ single crystal diamond platelet. A multiplex ICP etch tool (SPTS) was used to transfer the PR mask to diamond. Based on the study shown in Sec. II B, for a PR microlens of $400 \mu \mathrm{m}$ in diameter and a reflow temperature of $125^{\circ} \mathrm{C}$, the minimum PR thickness is around $17 \mu \mathrm{m}$, which is beyond the thickness achievable using a double PR layer deposition. Furthermore, increasing the PR thickness will also result in significantly reduced uniformity and pattern fidelity due to an increasing influence of the edge bead effect on the small diamond sample. Therefore, the reflow temperature was raised to $135^{\circ} \mathrm{C}$, which allowed PR microlens formation with a modified initial thickness of $\mathrm{h}_{0}=11.5 \mu \mathrm{m}$ on diamond with high pattern fidelity. By this way, the resulting PR microlens on diamond had a ROC as large as $1.4 \mathrm{~mm}$. To fabricate a diamond microlens with a larger ROC, an etch recipe with a low etch selectivity, which is defined by the ratio of diamond etch rate divided by the PR etch rate, is required to transfer the $\mathrm{PR}$ microlens mask to diamond.

In this work, transferring the PR lens mask to diamond is done by ICP etching with $\mathrm{Ar} / \mathrm{Cl}_{2}$ plasma. ${ }^{16}$ The chlorinebased diamond etching has a very low etch selectivity of 0.1 , nearly half that of the conventional $\mathrm{Ar} / \mathrm{O}_{2}$ etching under the same conditions. ${ }^{16}$ Therefore, it enables a much shallower etched diamond structure and a larger ROC of resulting diamond microlenses. The etch recipe used here was: $5 \mathrm{mTorr}$ pressure, $25 \mathrm{sccm}$ Ar flow rate, $40 \mathrm{sccm} \mathrm{Cl}_{2}$ flow rate, $300 \mathrm{~W}$ platen power, and $400 \mathrm{~W}$ coil power.

\section{B. Characterization of large-ROC diamond microlenses}

As measured by the atomic force microscopy, the fabricated diamond microlenses have a root-mean-square surface roughness of only $0.18 \mathrm{~nm}$ for an area of $3 \times 3 \mu \mathrm{m}^{2}$. This result is consistent with the roughness previously reported by Lee et al. ${ }^{16}$ Such a smooth surface is beneficial for the optical applications. To characterize the profiles of the fabricated diamond microlenses, a Dektak surface profilometer was used. The height of the fabricated microlenses was measured to be around $1.6 \mu \mathrm{m}$. By fitting with a semicircle function, a ROC value of $14 \mathrm{~mm}$ is obtained, as shown in Fig. 7. An optical image of the fabricated diamond

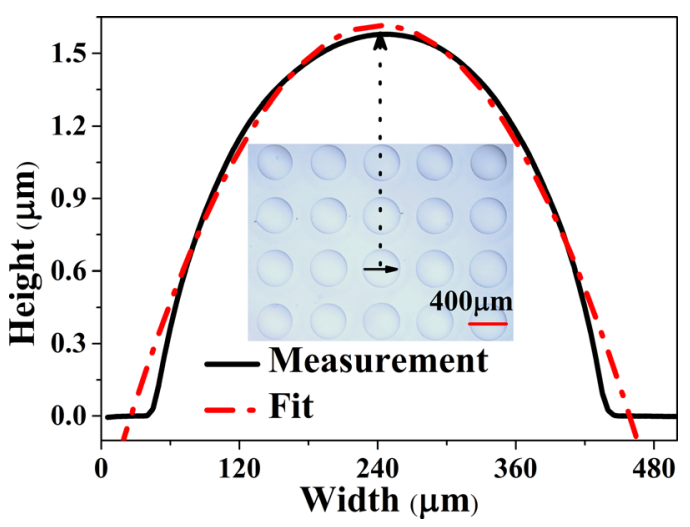

Fig. 7. (Color online) Profile of a diamond microlens including a spherical fit. Inset: optical image of the diamond microlens array. 
microlenses is also presented in Fig. 7 as the inset. A deviation from the spherical shape is observed at the profile bottom, which comes from the large ROC PR microlens and the low-selectivity ICP etching. If the lens shape were perfectly spherical, then the ROC would be slightly smaller, namely, $13 \mathrm{~mm}$, as calculated with Eq. (4).

\section{Outlook}

Diamond microlenses with such large ROCs can be used to form microresonators and serve as crucial components for the application in a monolithic diamond Raman laser. ${ }^{13,14}$ The technique of diamond microlens fabrication with a special geometry might also find use in solid-immersion-lenses for quantum related technologies. ${ }^{10,29,30}$ Similarly, microlenses with different diameters and ROCs on various materials can be achieved following the principles outlined here on demand and might find their application in imaging ${ }^{31}$ or displays. ${ }^{32}$ Furthermore, based on this study, shapes other than spherical convex lenses, such as microrings, ${ }^{33}$ concavelenses, and aspheric microlenses, are also achievable by the precise control of the PR reflow.

\section{CONCLUSIONS}

The edge bulge effect exhibited during the PR pattern reflow process has been studied, and the findings have been applied to develop novel diamond microlenses. An empirical description and relationship of the PR edge bulge evolution as a function of the initial PR thickness and the reflow temperature are provided with the underlying physics still open to being discussed. Importantly, at certain pattern diameters, we observed a minimum PR thickness at which a spherical PR lens structure can be formed by thermal reflow and the lens profile formed has the largest ROC. This minimum thickness is predictable by using Eq. (1). This study underpins the controllable fabrication of PR patterns using thermal reflow. Furthermore, by combining the controllable fabrication of PR patterns with the PR pattern transfer via ICP etching, the diamond microlenses with a large ROC have been achieved. In particular, pattern transfer through $\mathrm{Ar} / \mathrm{Cl}_{2} \mathrm{ICP}$ etching takes advantage of a low etch selectivity and enables subnanometer surface roughness. Microlenses with a diameter of $400 \mu \mathrm{m}$ were fabricated in this work have a ROC of $1.4 \mathrm{~mm}$ in PR and over $13 \mathrm{~mm}$ in diamond after pattern transfer. Such large ROC lenses have not been achieved previously and have wide range of applications.

\section{ACKNOWLEDGMENTS}

The author acknowledges support from the China Scholarship Council for the studentship (File No. 201206950018). Thanks to Element 6 for the supply of diamond samples. The authors thank Ross Leyman and the James Watt Nanofabrication Centre for helping with the fabrication of some samples. Thanks also to Enyuan Xie and Sean Reilly for helpful discussions.

${ }^{1}$ D. Daly, R. F. Stevens, M. C. Hutley, and N. Davies, Meas. Sci. Technol. 1, 759 (1990).

${ }^{2}$ H. W. Choi, E. Gu, C. Liu, C. Griffin, J. M. Girkin, I. M. Watson, and M. D. Dawson, J. Vac. Sci. Technol., B 23, 130 (2005).

${ }^{3}$ E. Roy, B. Voisin, J. F. Gravel, R. Peytavi, D. Boudreau, and T. Veres, Microelectron. Eng. 86, 2255 (2009).

${ }^{4}$ H. W. Choi, E. Gu, C. Liu, J. M. Girkin, and M. D. Dawson, J. Appl. Phys. 97, 063101 (2005).

${ }^{5}$ H. W. Choi, C. Liu, E. Gu, G. McConnell, J. M. Girkin, I. M. Watson, and M. D. Dawson, Appl. Phys. Lett. 84, 2253 (2004).

${ }^{6}$ E. Gu, H. W. Choi, C. Liu, C. Griffin, J. M. Girkin, I. M. Watson, M. D. Dawson, G. McConnell, and A. M. Gurney, Appl. Phys. Lett. 84, 2754 (2004).

${ }^{7}$ N. Laurand, C. L. Lee, E. Gu, J. E. Hastie, S. Calvez, and M. D. Dawson, Opt. Express 15, 9341 (2007).

${ }^{8}$ G. E. Harlow, The Nature of Diamonds (Cambridge University, Cambridge, 1998).

${ }^{9}$ V. G. Savitski, S. Reilly, and A. J. Kemp, IEEE J. Quantum Electron. 49, 218 (2013).

${ }^{10}$ J. P. Hadden, J. P. Harrison, A. C. Stanley-Clarke, L. Marseglia, Y. L. D. Ho, B. R. Patton, J. L. O’Brien, and J. G. Rarity, Appl. Phys. Lett. 97, 241901 (2010).

${ }^{11}$ S. Castelletto et al., New J. Phys. 13, 025020 (2011).

${ }^{12}$ N. Laurand, C. L. Lee, E. Gu, S. Calvez, and M. D. Dawson, IEEE Photonics Technol. Lett. 21, 152 (2009).

${ }^{13}$ H. Liu, S. Reilly, J. Herrnsdorf, E. Xie, V. G. Savitski, A. J. Kemp, E. Gu, and M. D. Dawson, Diamond Relat. Mater. 65, 37 (2016).

${ }^{14}$ S. Reilly, V. G. Savitski, H. Liu, E. Gu, M. D. Dawson, and A. J. Kemp, Opt. Lett. 40, 930 (2015).

${ }^{15}$ Z. D. Popovic, R. A. Sprague, and G. A. Connell, Appl. Opt. 27, 1281 (1988).

${ }^{16}$ C. L. Lee, E. Gu, M. D. Dawson, I. Friel, and G. A. Scarsbrook, Diamond Relat. Mater. 17, 1292 (2008).

${ }^{17}$ F. T. O’Neill and J. T. Sheridan, Optik 113, 391 (2002).

${ }^{18}$ S. Audran, B. Mortini, B. Faure, and G. Schlatter, J. Micromech. Microeng. 20, 095008 (2010).

${ }^{19}$ A. Schilling, R. Merz, C. Ossmann, and H. P. Herzig, Opt. Eng. 39, 2171 (2000).

${ }^{20}$ R. D. Deegan, O. Bakajin, T. F. Dupont, G. Huber, S. R. Nagel, and T. A. Witten, Nature 389, 827 (1997).

${ }^{21}$ S. Das, P. R. Waghmare, M. Fan, N. S. K. Gunda, S. S. Roy, and S. K. Mitra, RSC Adv. 2, 8390 (2012).

${ }^{22}$ T. Dobroth and L. Erwin, Polym. Eng. Sci. 26, 462 (1986).

${ }^{23}$ I. S. McKinley and S. K. Wilson, Phys. Fluids 14, 133 (2002).

${ }^{24}$ E. Woerner, C. Wild, W. Mueller-Sebert, and P. Koidl, Diamond Relat. Mater. 10, 557 (2001).

${ }^{25}$ N. Laurand, C. L. Lee, E. Gu, J. E. Hastie, A. J. Kemp, S. Calvez, and M. D. Dawson, IEEE J. Quantum Electron. 44, 1096 (2008).

${ }^{26}$ N. Ph, R. Völkel, H. P. Herzig, M. Eisner, and S. Haselbeck, Pure Appl. Opt. 6, 617 (1997).

${ }^{27}$ B. Lautrup, Physics of Continuous Matter: Exotic and Everyday Phenomena in the Macroscopic World (Institute of Physics, London, 2004).

${ }^{28}$ F. T. O'Neill and J. T. Sheridan, Optik 113, 405 (2002).

${ }^{29}$ M. Jamali, I. Gerhardt, M. Rezai, K. Frenner, H. Fedder, and J. Wrachtrup, Rev. Sci. Instrum. 85, 123703 (2014).

${ }^{30}$ W. Pfaff et al., Science 345, 532 (2014).

${ }^{31}$ R. P. Rocha, J. P. Carmo, J. M. Gomes, M. Belsley, and J. H. Correia, Procedia Eng. 47, 619 (2012).

${ }^{32}$ T. Knieling, M. Shafi, W. Lang, and W. Benecke, J. Eur. Opt. Soc. 7, 12007 (2012).

${ }^{33}$ M. H. Wu, K. E. Paul, and G. M. Whitesides, Appl. Opt. 41, 2575 (2002). 